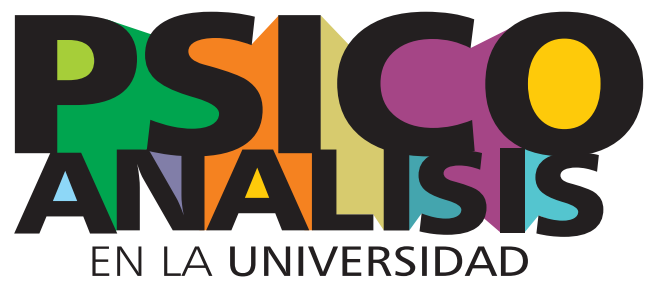

Departamento de Psicoanálisis FACULTAD DE PSICOLOGÍA, UNR

\title{
Breve reseña curricular de quienes integran la dirección y el comité editorial
}

\section{DIRECTORA}

\section{Silvina Garo}

Psicoanalista. Magister en Psicoanálisis, UNR. Profesora Adjunta de la Cátedra Psicoanálisis y psicopatología. Coordinadora Académica del Área de Publicaciones de la Facultad de Psicología. Miembro del Comité Académico de la Maestría Clínica con Niños. Actualmente Directora del Departamento de Psicoanálisis de la Facultad de Psicología, UNR. Docente-investigadora categorizada, Evaluadora y directora de tesis en la carrera de Maestría en Psicoanálisis. Coordinadora general de las Jornadas bianuales Analistas hablando de su práctica, Facultad de Psicología, UNR.

Ha publicado su primer libro Navegar es preciso. Una travesía por la clínica freudiana a través del duelo (Homo Sapiens; 2015) y numerosos artículos en diversas revistas y libros de interés científico: Revista Psicoanálisis en la Universidad, (UNR editora); Historización y duelo, Revista Psicoanálisis y el Hospital N43; Revista Universitaria de Psicoanálisis, Año 2013 (Facultad de Psicología, UBA); La fábrica de casos II: los personajes de Ernesto Sábato (Homo Sapiens;1999); Argumentos 6, Revista de la Escuela Sigmund Freud (Rosario; 2001). garosilvina@gmail.com

\section{CO-DIRECTOR}

\section{Jorge Ceballos}

Psicoanalista. Psicólogo. Doctorando en Psicología, Facultad de Psicología. UNR.

Docente e investigador categorizado, y Jefe de trabajos prácticos concursado de la materia Psicoanálisis I de la Facultad de Psicología, UNR. Ha dictado un Seminario de Pregrado en Fac. Psicología (UNR) así como ha sido Titular de la Cátedra PsicologíaGeneral en Licenciatura en Kinesiología y Fisiatría (IUGR). Es autor de ponencias en jornadas y congresos, así como de diversas publicaciones. Ha sido Jurado de concurso y de tesis. Es miembro del equipo interdisciplinario y co-coordinador del Programa para Jóvenes en el C.E.T. “Un Lugar para Aprender". Fundación del Centro del Desarrollo Infantil.

Ha publicado artículos en El sujeto en la experiencia analítica. Segundas jornadas de intercambio UNR Editora (2000), Cuadernos de Metapsicología. Cátedra Psicoanálisis I. UNR Editora ( $N^{\circ} 4,2013$ ), Actas de jornadas de investigación. Facultad de Psicología.UNR. (2016, 2015, 2014, 2013), en el libro Caleidoscopio, prácticas y clínicas Psi en la Universidad. Faccendini y otros (comp.) UNR Editora. (2018) y en la Revista Psicología Digital 
(2018). Revista del Programa Problemáticas Contemporáneas. Psicoanálisis, Ciencia, Ciencia Cognitiva, CEI- UNR.

jorgecceballos@gmail.com

\section{COMITÉ EDITORIAL}

\section{Bruno Carignano}

Psicoanalista. Magister en psicoanálisis. Doctorando en Cotutela por convenio entre la Université Paris Diderot - ComUE Sorbonne Paris Cité, Centre de recherches psychanalyse, médecine et société (CRPMS) - y la Universidad Nacional de Rosario, Facultad de Psicología, Secretaría de Estudios de Posgrados, Doctorado en Psicología.

Es docente e investigador categorizado, ha sido becario de diversos programas para la formación académica en el país y en el exterior. Se desempeña actualmente como Jefe de Trabajos Prácticos concursado en la cátedra Psicoanálisis II de la Facultad de Psicología de UNR y como Profesor Titular concursado en UCES, en las cátedras Psicoanálisis Freud II, Psicoanálisis Escuela Francesa, Taller de Trabajo Integrador Final y Psicopatología II. Es actualmente director de una investigación en curso en UCES y ha participado como integrante en diversas investigaciones radicadas en la UNR. Es autor de diversas ponencias en Jornadas y Congresos y ha publicado numerosos artículos y traducciones en revistas de Psicoanálisis y Psicología.

Se ha desempeñado como director y evaluador de Trabajos de Integración Final en la formación de Grado de la Facultad de Psicología de la UNR y UCES, y como codirector de tesis de maestría. Participa, además, como referato de la revista de Psicología Wimb-Lu (Costa Rica).

brunocarignano@yahoo.com

\section{Valeria Decorte}

Psicoanalista, Magister en Psicoanálisis: Tesis: "Los silencios en Psicoanálisis. Distintos estatutos del silencio en la clínica". Docente e investigadora categorizada. Jefe de trabajos prácticos en la materia Clínica II. Docente del "Taller de investigación y escritura II" en la Maestría en Psicoanálisis de la Facultad de Psicología de la UNR. Directora, co-directora, evaluadora y jurado de tesis en dicha carrera. valeriadecorte@gmail.com

\section{Olivia Di Nardo}

Psicóloga por la UNR, Psicoanalista, practicante del psicoanálisis con niños. Participante de la Escuela de psicoanálisis Sigmund Freud de Rosario. Secretaria del Departamento de Psicoanálisis de la Facultad de Psicología UNR. JTP de la cátedra Psicoanálisis II.

Investigadora. Miembro del equipo interdisciplinario Diálogos Rosario.

Ha participado como expositora de diversos congresos nacionales e internacionales.

oliviadinardo@yahoo.com.ar

\section{Antonio S. Gentile}

Psicoanalista; se tituló de grado como Psicólogo, en la Escuela Superior de Psicología, de la UNR. Profesor Titular ordinario, desde 1987. Investigador Principal de la Carrera de Investigador Científico de la UNR (CIC-UNR). Doctor de la UNR, con una tesis sobre el concepto de memoria en la obra de Sigmund Freud. Docente-investigador categoría I.

Ha publicado artículos en revistas científicas nacionales y extranjeras, ha contribuido en numerosos congresos nacionales e internacionales. Autor de tres libros, uno de ellos en colaboración con el equipo de la cátedra de 
"Psicología". Dirige proyectos de investigación psicoanalíticos y tesis a nivel de Maestría en Psicoanálisis y Doctorado.

Ha dictado cursos de posgrado en las universidades nacionales del Comahue, Mar del Plata, La Plata y Rosario y en universidades de Brasil y Colombia e instituto especializado en Israel. Ha sido director de la Carrera de Doctorado de la Facultad de Psicología-UNR y miembro titular de la Comisión de Pos-Doctorado de la UNR. Con la recuperación de la democracia, se comprometió con la sanción de las leyes del ejercicio profesional y la organización social de los psicólogos, a nivel local y nacional. Integra en Grupo de Trabajo en Historia de la Psicología, de la Sociedad Interamericana de Psicología. Es miembro del Comité Asesor Nacional del Centro Argentino de Historia del Psicoanálisis, la Psicología y la Psiquiatría de la Biblioteca Nacional.

antoniogentile.rosario@gmail.com

\section{Cecilia Gorodischer}

Psicoanalista. Psicóloga por la Facultad de Psicología de la UNR. Magíster por la Facultad Latinoamericana de Ciencias Sociales (FLACSO). Doctora en Psicología (UNR).

Investigadora Independiente de la Carrera de la Investigación Científica del Consejo de Investigaciones (CIC-CIUNR). Docente -Investigadora Categoría II. Profesora Adjunta de la Facultad de Psicología (UNR).

Directora de tesis doctorales y de maestría. Directora de numerosos Proyectos de Investigación (1990 y continúa). Actualmente dirige el Proyecto de Investigación titulado "Formulaciones actuales sobre el cuerpo en Psicoanálisis. Sus incidencias clínicas". Directora del Programa Interdisciplinario en Psicoanálisis, Enseñanza y Transmisión (PIPET), radicado en el Instituto de Estudios Críticos en Humani- dades (IECH-CONICET) y en el Centro de Estudios Interdisciplinarios de la UNR (CEI-UNR). Posdoctora en la Universidad Nacional de Rosario. Publicó en numerosas revistas científicas.

cgorodischer@gmail.com

\section{Cecilia Greca}

Psicóloga, Profesora en Psicología y Magíster en Psicoanálisis por la Universidad Nacional de Rosario. Psicoanalista. Jefe de Trabajos Prácticos concursada en la cátedra de Psicología de la Facultad de Psicología de la UNR. Investigadora categorizada y directora del proyecto de extensión universitaria titulado "Palabras que cuentan" (2020-2021). Ha sido becaria de diversos programas para la formación académica en el exterior.

Ha integrado jurados de tesis de Maestría en la Facultad de Psicología de la UNR.

Integrante del equipo del Dispositivo Integral de Abordaje Territorial (DIAT Rosario) como profesional dependiente del Ministerio de Desarrollo Social de la Provincia de Santa Fe. Autora del libro La contratransferencia: ¿asunto superado o asunto clausurado? Un recorrido desde Freud y Lacan, de Laborde Editor (2018) y coautora de los libros La Psicología en el diván. Ensayos críticos sobre el saber psicológico (2009) y Psicoanálisis por estudiantes. Un recorrido por las nuevas formas de hacer Universidad (2008), ambos de UNR Editora. Ha sido expositora en numerosos congresos, simposios y jornadas, tanto nacionales como internacionales.

cecigreca@gmail.com 


\section{Soledad Nívoli}

Doctora en Ciencia Política (UNR). Magister en Literatura Argentina (UNR). Psicóloga y Profesora de Psicología (UNR).

Ha realizado estudios de Posdoctorado en la UNGS (Los Polvorines, Bs. As.) y en la Universidad de París 8 (Saint Denis, Francia). Es Directora del Centro de Estudios Periferia Epistemológica (CEPE-UNR) y de la Biblioteca Abierta «La Cita Rosa». Es miembro del Laboratoire Tournant (Paris 8). Docente e investigadora de la Facultad de Psicología de la UNR y del IUNIR. Sus temas de investigación cruzan los campos del psicoanálisis, le teoría literaria, la teoría política y la filosofía. Ha prologado y traducido Sublevarse (entrevista inédita a Michel Foucault, Catálogo, Viña del Mar, 2016) y El Tercer Reich de los sueños de Charlotte Beradt (junto a Leandro Levi, LOM, Santiago de Chile, 2019). Actualmente se encuentra realizando una investigación sobre la elaboración onírica en tiempos del COVID-19, junto a la Dra. Lucía Brienza y a la Ps. Flavia Castro.

lanivo@yahoo.com.ar

\section{Andrés Palavecino}

Psicólogo por la Universidad Nacional de Rosario. Es jefe de trabajos prácticos en la asignatura Clínica 1, de la Facultad de Psicología U.N.R. Docente auxiliar a cargo del Seminario de Pregrado "Materia de Lectura" de la Facultad de Psicología U.N.R Escribe, investiga, edita, crea contenidos y proyectos en el campo psicoanálisis y la filosofía. Su múltiple experiencia en diversos ámbitos -culturales, hospitalarios, editoriales- le permite trabajar cómodamente en equipo transdisciplinar. Su proactividad le posibilita producir contenidos en diversos formatos -materiales, simbólicos, conceptuales, experimentales, proyectuales-. Es uno de los editores de Otro cauce, editorial de psicoanálisis y filosofía. andres.plvcn@gmail.com 